\title{
Engineered noisy environment for studying decoherence
}

\author{
Ai Iwakura, ${ }^{1}$ Yuichiro Matsuzaki, ${ }^{2}$ and Yasushi Kondo ${ }^{1,3, *}$ \\ ${ }^{1}$ Interdisciplinary Graduate School of Science and Engineering, Kindai University, Higashi-Osaka 577-8502, Japan \\ ${ }^{2}$ NTT Basic Research Laboratories, NTT Corporation, Kanagawa 243-0198, Japan \\ ${ }^{3}$ Department of Physics, Kindai University, Higashi-Osaka 577-8502, Japan
}

(Received 6 November 2016; published 1 September 2017)

\begin{abstract}
The largest obstacle to perform quantum information processing is decoherence of a system. In order to overcome this, various techniques, such as dynamical decoupling and quantum Zeno effects, have been proposed and demonstrated. Here, we present an NMR model with which various decoherence suppression techniques can experimentally be evaluated. By changing the conditions in the sample preparation, we can engineer an environment to interact the system that contains the information. Moreover, we can efficiently describe the dynamics by the operator-sum representation due to the simplicity of our model. As concrete examples, we have investigated the performance of dynamical decoupling with several molecules. Our model provides a useful test bench to understand the mechanism of decoherence induced by a noisy environment and to examine various ideas of decoherence suppression techniques.
\end{abstract}

DOI: 10.1103/PhysRevA.96.032303

\section{INTRODUCTION}

Control of quantum systems can in principle provide many useful applications such as quantum computation, quantum cryptography, quantum simulation, and quantum metrology [1]. One of the largest obstacles against the realization of quantum applications is decoherence of a system. We need to advance our understanding of the decoherence mechanism [1-11] and develop suppression techniques [12-14] for reliable quantum controls.

Decoherence is considered as irreversible changes of a state of a quantum system due to the coupling between the system and environment. When decoherence occurs, there is a correlation between the system and the environment. Since we cannot track all information that environment has, we loose some information about the coupled system, which results in the irreversible changes [1-5,11]. Therefore, in order to understand decoherence, it is necessary to answer the following questions: How is the system interacted by the environment? How does the environment lose the information by time development? Some decoherence dynamics can be described by an autocorrelation function that accounts for the correlation between the system and environment [15]. An idealized boson-bath model [16] has been also frequently employed in theoretical works in order to evaluate its dynamics. Microscopic models are also useful to consider the decoherence suppression techniques, and this kind of analysis has been done in several systems such as a quantum dot $[17,18]$.

On the other hand, various engineered noises have been proposed and employed for understanding decoherence and evaluating techniques against it [12-14]. Such techniques include dynamical decoupling (DD), quantum error corrections, and quantum Zeno effects [19-23]. The following are examples of engineered noises. A spin embedded in solid (often ${ }^{13} \mathrm{C}$ ) is regarded as a system and the other spins (often ${ }^{1} \mathrm{H}$ ) surrounding the system consist of its environment [24-26]. Some other spins in a molecule that contains a spin acting as a

\footnotetext{
*ykondo@kindai.ac.jp
}

system may be regarded as a measurement device [27] in NMR experiments. Note that decoherence can be considered as a result of a measurement of the system by the environment [28]. Furthermore, a combination of a field gradient application with other methods such as molecular diffusion [29,30] or ensemble averaging over the sample [31] can be employed for generating dephasing. It is worth mentioning that a noisy external field can also be introduced by modulating this external field itself [32] or equivalently, by modulating a reference signal $[33,34]$ with which measurements are synchronized. Interestingly, we could combine the random classical fields with a small number of spins, which can simulate an environment [35] where the phase decoherence of the system in the above simulated environment is suppressed by DD [36,37]. After an engineered coupling is introduced by applying a well-controlled external field, the effect of this coupling is removed by DD [38], by quantum error correction procedures $[39,40]$, or by quantum Zeno effects [41].

However, in the most of the previous works, the noise has been induced via active manipulations (such as pulse implementations) by the experimentalists. The environment generated with such active operations by the experimentalists is rather artificial and could be called a simulated environment. Such an approach is usually time consuming due to the requirement of many repetitions to simulate noise. Also, active operations to simulate the decoherence tend to induce unexpected noise unless the experimentalists have perfect controls. There are only few works that actually engineer the environment itself $[26,42]$. In these experiments, the concentrations of noise sources in the samples were controlled. Since no active operations are required after preparing the sample, we would call this approach a control of the real environment.

The purpose of this work is to introduce a NMR model for studying decoherence and its suppression technique by engineering a real environment to induce noise when the sample is prepared. We combine the methods in $[27,35,43]$ with the scheme in [42] to realize our model. The system in our model is a single spin in a molecule while the environment consists of the other spins in the same molecule 
and magnetic impurities in the sample solvent. Since we can select the molecules of which internal interactions (or, equivalently, its structure) are well known [44,45], we can easily understand the dynamics between the system spin and environmental spins. On the other hand, the dynamics between the environmental spins and the magnetic impurities can be modeled by a stochastic flip-flopping motion of the spins or random telegraph noise [46]. Due to both experimental feasibility and the simplicity of the theoretical framework, our model provides us with a useful test bench to understand the mechanism of the decoherence and to evaluate various techniques against decoherence.

The rest of the paper is organized as follows. In Sec. II, we have a brief review of the theory of a quantum channel, which is a useful formalism to describe decoherence in a general context. We apply this concept of quantum channel to understand our NMR model so that we can confirm that DD, one of the examples of decoherence suppression techniques, is effective for the model. In Sec. III, we experimentally confirm our capability to engineer the environment and the validity of DD to suppress the decoherence for our model. For this purpose, we use the standard pulse sequence for the demonstration of DD and also use the standard theory to analyze the experimental data. In Sec. IV, we perform numerical simulations that include imperfect pulse operations in DD and compare the results with the experiments. Section $\mathrm{V}$ is devoted to a summary and conclusions.

\section{UNDERSTANDING THE MODEL WITH A QUANTUM CHANNEL}

A system with magnetic impurities has been an important issue in the history of NMR and has extensively been investigated in the NMR community [47]. Instead of employing the modeling developed in the NMR community, we here describe the system in terms of a quantum channel which is known to be compatible with quantum information processing (QIP), because our primary interest is its application to QIP.

\section{A. Quantum channel}

Let $\mathscr{H}_{\mathrm{S}}$ and $\mathscr{H}_{\mathrm{E}}$ be the Hilbert spaces of the system and the environment, respectively. The initial state of the system (environment) is represented by the density matrix $\rho_{\mathrm{S}}\left(\rho_{\mathrm{E}}\right)$. The state of the whole system changes as follows:

$$
\rho_{\mathrm{S}} \otimes \rho_{\mathrm{E}} \rightarrow U \rho_{\mathrm{S}} \otimes \rho_{\mathrm{E}} U^{\dagger}
$$

Here $U$ is a unitary operator acting on the whole Hilbert space $\mathscr{H}_{\mathrm{S}} \otimes \mathscr{H}_{\mathrm{E}}$ and we assume that the initial state is separable. If we are only interested in the state of the system, the measurement outcomes are completely described by the reduced density matrix

$$
\rho_{\mathrm{S}}^{\prime}=\mathscr{E}\left(\rho_{\mathrm{S}}\right)=\operatorname{Tr}_{\mathrm{E}}\left(U \rho_{\mathrm{S}} \otimes \rho_{\mathrm{E}} U^{\dagger}\right),
$$

where $\operatorname{Tr}_{\mathrm{E}}$ denotes the partial trace over $\mathscr{H}_{\mathrm{E}}$. The number of the degree of freedom of $\mathscr{H}_{\mathrm{E}}$ is assumed to be infinite and thus the information flow from the system to the environment is one way. In this case, a trace operation is not reversible and the associated loss of information leads to decoherence.
The mapping $\rho_{\mathrm{S}} \mapsto \mathscr{E}\left(\rho_{\mathrm{S}}\right)$ is called a channel or a quantum operation $[1,48,49]$. It is known that, for a channel, there is a set of operators $\left\{E_{k}\right\}$ acting on $\mathscr{H}_{\mathrm{S}}$ such that

$$
\begin{gathered}
\mathscr{E}\left(\rho_{\mathrm{S}}\right)=\sum_{k} E_{k} \rho_{\mathrm{S}} E_{k}^{\dagger}, \\
\sum_{k} E_{k}^{\dagger} E_{k}=I_{\mathrm{S}} .
\end{gathered}
$$

Here $I_{\mathrm{S}}$ is the identity operator on $\mathscr{H}_{\mathrm{S}}$. Equation (3) is called an operator-sum representation of the channel $\mathscr{E}$. Equation (4) implies $\operatorname{Tr}_{\mathrm{S}} \mathscr{E}\left(\rho_{\mathrm{S}}\right)=\operatorname{Tr}_{\mathrm{S}} \rho_{\mathrm{S}}, \operatorname{Tr}_{\mathrm{S}}$ being the trace over $\mathscr{H}_{\mathrm{S}}$ and hence it is called the trace-preserving condition.

\section{B. A few-spin system}

Let us consider a few-spin system isolated from the environment. We divide this system into two subsystems, one spin (called subsystem s) and the other spins (called subsystem e). The total Hamiltonian is decomposed as

$$
H=H_{\mathrm{s}}+H_{\mathrm{e}}+H_{\mathrm{se}} .
$$

Here $H_{\mathrm{s}}$ and $H_{\mathrm{e}}$ govern intrinsic behaviors of subsystems s and e, while $H_{\mathrm{se}}$ describes an interaction between them.

It is worth mentioning that subsystem e with the small number of freedom can simulate an open environment by averaging measurement results where external stochastic fields are randomly applied [35].

Although such an artificial environment or a bathengineering technique by modulating control field has a large degree of freedom that experimentalists can control, there are at least two major drawbacks. First, such a large degree of freedom inevitably induces additional unexpected noise unless we have a perfect control of all freedom. Second, the artificial environment approach is much more time consuming than our approach, because the nonunitary properties are given by averaging many experimental runs in the case of the artificial environment approach. For example, as many as 128 runs were averaged in our previous work [36], while a single run is required in our approach presented in this work.

In this paper, we introduce a well-controlled real environment (called system E) by adding magnetic impurities. This environment is significantly coupled with subsystem e, while it has a negligible coupling with subsystem $\mathrm{s}$. The total Hamiltonian is now given as

$$
H=H_{\mathrm{s}}+H_{\mathrm{e}}+H_{\mathrm{se}}+H_{\mathrm{E}}+H_{\mathrm{eE}},
$$

where $H_{\mathrm{E}}$ denotes an intrinsic behavior of system $\mathrm{E}$ and $H_{\mathrm{eE}}$ denotes an interaction between subsystem e and system $\mathrm{E}$. It is worth mentioning that the term $H_{\mathrm{E}}+H_{\mathrm{eE}}$ naturally generates stochastic random fields on subsystem e, which corresponds to randomly applied pulses in our previous experiments [36].

Also, we can rewrite this Hamiltonian as follows:

$$
H=H_{\mathrm{s}}+H_{\mathrm{sE}^{\prime}}+H_{\mathrm{E}^{\prime}},
$$

where $H_{\mathrm{E}^{\prime}}=H_{\mathrm{e}}+H_{\mathrm{E}}+H_{\mathrm{eE}}$ and $H_{\mathrm{sE}^{\prime}}=H_{\mathrm{se}}$. Here, we have a well-defined environment $\mathrm{E}^{\prime}$. We also have the interaction between the environment $\mathrm{E}^{\prime}$ and system s. Importantly, we can control $H_{\mathrm{E}^{\prime}}$ by tuning an amount of magnetic impurities in our samples and by selecting a molecule. This technique has been 


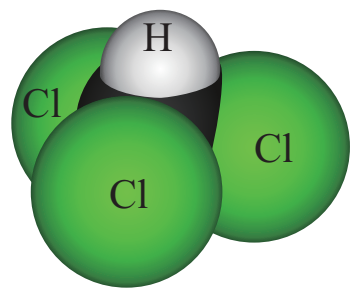

FIG. 1. Chloroform molecule. The center black sphere is a carbon atom which is surrounded by one hydrogen atom and three chlorine atoms. Due to the surrounding atoms, the carbon atom is not directly influenced by magnetic impurities.

applied for a principle-of-proof experiment of the quantum Zeno effect [50]. We show its other application, or evaluating $\mathrm{DD}$, in this work.

\section{Chloroform molecule as a few-spin system}

We show our idea with a ${ }^{13} \mathrm{C}$-labeled chloroform $\left(\mathrm{CHCl}_{3}\right)$ molecule as a few-spin (qubit) system. Chloroform molecules are solved in acetone-d6 where magnetic impurities of Fe(III) acetylacetonate are also added. The Fe(III) ions with strong dipole magnetic moments move randomly in the solution and create external stochastic fields for chloroform molecules when $\mathrm{Fe}(\mathrm{III})$ ions approach them. The spins in the chloroform molecule are controlled by using the NMR technique. We consider the ${ }^{13} \mathrm{C}$ spin as a subsystem s, while the ${ }^{1} \mathrm{H}$ spin is considered as a subsystem e, see Fig. 1 . The ${ }^{13} \mathrm{C}$ atom is surrounded by the ${ }^{1} \mathrm{H}$ and $\mathrm{Cl}$ atoms, and the $\mathrm{Cl}$ atoms are magnetically inactive. Therefore, we can approximate that the ${ }^{13} \mathrm{C}$ spin is not directly influenced by the external stochastic field but is indirectly affected through the ${ }^{1} \mathrm{H}$ spin.

We take a rotating frame for the ${ }^{13} \mathrm{C}\left({ }^{1} \mathrm{H}\right)$ spin of which angular velocity is its Larmor frequency. In this rotating frame, $H_{\mathrm{s}}\left(H_{\mathrm{e}}\right)$ in Eq. (6) vanishes. $H_{\mathrm{se}}$ of the chloroform molecule is given as

$$
H_{\mathrm{se}}=\frac{J}{4} \sigma_{z} \otimes \sigma_{z},
$$

where $J$ denotes the strength of the interaction between the subsystem s (the ${ }^{13} \mathrm{C}$ spin) and the subsystem e (the ${ }^{1} \mathrm{H}$ spin). We will consider the effect of $H_{\mathrm{eE}}$ (the interaction between the ${ }^{1} \mathrm{H}$ spin and environment) in Eq. (6) by using an operator-sum formalism as described in the following subsection.

\section{Noise channel}

We simulate the spin dynamics in chloroform molecules with an operator-sum formalism. $H_{\mathrm{se}}$ leads to the time development of the total density matrix $\rho$ as

$$
A d\left(e^{-i H_{\mathrm{se}} t}, \rho\right),
$$

where $\operatorname{Ad}(*, \rho)=* \rho *^{\dagger}$. The decoherence process is determined by the magnetic impurities, and the time development of the density matrix of ${ }^{1} \mathrm{H}$ (the subsystem e), $\rho_{\mathrm{e}}$, in the time interval of $\delta$ is conveniently given as

$$
\mathscr{E}_{\mathrm{e}}\left(\rho_{\mathrm{e}}\right)=e^{-\kappa \delta} \rho_{\mathrm{e}}+\left(1-e^{-\kappa \delta}\right) X(\theta) \rho_{\mathrm{e}} X(\theta)^{\dagger},
$$

where we use a concept of a noise channel. Here $X(\theta)=$ $\sigma_{x} \cos \theta+\sigma_{y} \sin \theta$, and $\theta$ is a random valuable in $[0,2 \pi]$, and $\kappa$ determines a frequency of the flip-flop motion of the ${ }^{1} \mathrm{H}$ spin caused by magnetic impurities. The order of $\kappa$ is $1 / T_{\mathrm{e}}$, where $T_{\mathrm{e}}$ is a spin-spin relaxation time constant of the ${ }^{1} \mathrm{H}$ spin. Note that $\kappa$ is determined by $H_{\mathrm{eE}}$.

The time development of the total system during $\delta\left(\delta \ll T_{\mathrm{e}}\right)$ is

$$
\begin{aligned}
\rho(t+\delta)= & e^{-\kappa \delta} \operatorname{Ad}\left(e^{-i H_{\mathrm{se}} \delta}, \rho(t)\right) \\
& +\left(1-e^{-\kappa \delta}\right) A d\left(\left(\sigma_{0} \otimes X(\theta)\right) e^{-i H_{\mathrm{se}} \delta}, \rho(t)\right) \\
& \approx(1-\kappa \delta) A d\left(e^{-i H_{\mathrm{se}} \delta}, \rho(t)\right) \\
& +\kappa \delta A d\left(\left(\sigma_{0} \otimes X(\theta)\right) e^{-i H_{\mathrm{se}} \delta}, \rho(t)\right) .
\end{aligned}
$$

The time development of $\rho$ from $t=0$ to $T$ is obtained by

$$
\rho(0) \rightarrow \rho(\delta) \rightarrow \rho(2 \delta) \rightarrow \cdots \rightarrow \rho(T),
$$

where each step is determined by Eq. (9).

In our model, we can control and characterize timeinhomogeneous decoherence that has been paid attention by many researchers in the field of quantum information [5-11]. If the environment has a short memory time, the decoherence rate becomes time independent and so the decay process becomes exponential, which is called a time-homogeneous noise [51]. A system shows a time-inhomogeneous effect when its environment has a long memory time $[5,11]$. The effect of the second term in the Eq. (9) can be interpreted as the product of $e^{-i H_{\mathrm{se}} \delta}$ and $\sigma_{0} \otimes X(\theta)$, where $\kappa \delta$ denotes a probability. $e^{-i H_{\mathrm{se}} \delta}$ causes a memory effect of the environment [5]. In other words, the state of the ${ }^{1} \mathrm{H}$ spin does not change that of the ${ }^{13} \mathrm{C}$ spin immediately. On the other hand, $\sigma_{0} \otimes X(\theta)$ provides a mechanism to lose the memory of the environment because of its randomness. These two mechanisms that are necessary for time inhomogeneity are well separated in our model. This separation of two mechanisms is the unique point of our model compared with the boson-bath model [16]. We are able to control these mechanisms independently, as shown in Sec. III.

It is worth mentioning that the time-inhomogeneous noise is sometimes called non-Markovian noise [13]. This noise is not Markovian in the sense that it is not described by the standard Lindblad master equation proposed in [52]. However, we employ the term "time inhomogeneous" in this paper in order to avoid unnecessary confusion.

\section{Simulation of free induction decay}

The thermal density matrix of the chloroform molecule is approximated as

$$
\begin{aligned}
& \left(\frac{\sigma_{0}}{2}\right)^{\otimes 2}+\epsilon_{\mathrm{s}} \frac{\sigma_{z} \otimes \sigma_{0}}{4}+\epsilon_{\mathrm{e}} \frac{\sigma_{0} \otimes \sigma_{z}}{4} \\
& =\left(1-\epsilon_{\mathrm{s}}\right)\left(\frac{\sigma_{0}}{2}\right)^{\otimes 2}+\epsilon_{\mathrm{s}}|0\rangle\langle 0| \otimes \frac{\sigma_{0}}{2}+\epsilon_{\mathrm{e}} \frac{\sigma_{0} \otimes \sigma_{z}}{4},
\end{aligned}
$$

where the indices s and e denote ${ }^{13} \mathrm{C}$ and ${ }^{1} \mathrm{H}$, respectively. Also, $\epsilon_{i}=\hbar \omega_{i} / 2 k_{B} T$ denotes a ratio between the system energy and thermal energy, and $\omega_{i}$ is the Larmor frequency of the $i$ th spin. It is well known that the term $\left(1-\epsilon_{\mathrm{s}}\right)\left(\sigma_{0} / 2\right)^{\otimes 2}$ cannot be observed in NMR [45]. Moreover, we can ignore the third term $\epsilon_{\mathrm{e}} \sigma_{0} \otimes \sigma_{z} / 4$ if we do not measure the ${ }^{1} \mathrm{H}$ spin. Therefore, the above density matrix can be regarded as $\rho_{\text {th }}=\epsilon_{\mathrm{s}}|0\rangle\langle 0| \otimes \sigma_{0} / 2$.

The density matrix $\rho(t)$ after the operation ${ }^{\mathrm{s}}(\pi / 2)_{\pi / 2}$ applied on $\rho_{\text {th }}$ is calculated according to Eq. (9), where 


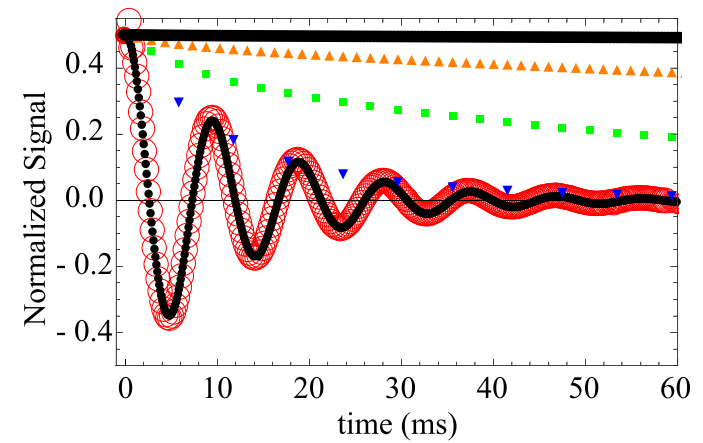

FIG. 2. Measured (O) and simulated (•) FID signals of the ${ }^{13} \mathrm{C}$ spin. The measured value of $J$ is $2 \pi \times 215.2 \mathrm{rad} / \mathrm{s}$. To reproduce the experiment, we choose a parameter of $1 / \kappa=13 \mathrm{~ms}$. Also, we use a value of $\delta=5.00 \times 10^{-2} \mathrm{~ms}$ in Eq. (9) for simulations. The relaxations, when DD pulses are applied, are also simulated. $\bullet, \wedge, \square$, and $\boldsymbol{\nabla}$ are results when $\tau_{\mathrm{b}}=0.2,0.8,1.6,3.2 \mathrm{~ms}$, respectively.

${ }^{i}(\theta)_{\phi}$ is a $\theta$ rotation of the $i$ th spin along the axis in the $x y$ plane of which the azimuthal angle is $\phi$. Then, the free induction decay (FID) signal of the ${ }^{13} \mathrm{C}$ spin is calculated by $\operatorname{Tr}_{\mathrm{e}}\left(\sigma_{x} \otimes \sigma_{0} \rho(t)\right)$. The simulation reproduces the experimental results very well when $1 / \kappa=13 \mathrm{~ms}$ is chosen, as shown in Fig. 2. The oscillatory behavior is caused by $H_{\mathrm{se}}$, while the decay of the signal amplitude (the envelope of the FID signal) indicates decoherence. Here, $1 / \kappa$ is twice that of the measured $T_{1}=T_{2}=7 \mathrm{~ms}$ of ${ }^{1} \mathrm{H}$ within experimental error.

\section{Dynamical decoupling in a few-spin system}

We discuss dynamical decoupling as one of the examples of decoherence suppression techniques in the case of a few-spin system. We adopt DD because this has been paid attention by many researchers with both theoretically and experimentally significant developments [12,22].

\section{Understanding DD based on quantum channel}

The dynamics of the ${ }^{13} \mathrm{C}$ spin of the chloroform molecule can be reversed by a pair of $\pi$ pulses assuming that the state of the ${ }^{1} \mathrm{H}$ spin remains unchanged, as shown below:

$$
\rho(t)=\operatorname{Ad}\left(e^{-i H_{\mathrm{se}} \delta_{1} \mathrm{~s}}(\pi)_{\pi} e^{-i H_{\mathrm{se}}\left(\delta_{1}+\delta_{2}\right) \mathrm{s}}(\pi)_{0} e^{-i H_{\mathrm{se}} \delta_{2}}, \rho(t)\right),
$$

where we can choose arbitrary values of $\delta_{1}$ and $\delta_{2}$. It is worth mentioning that we treat the case of the chloroform molecule as a few-spin system in order to make our discussion concrete.

In our case, the state of the ${ }^{1} \mathrm{H}$ spin, however, is not stationary because magnetic impurities move. Therefore, a single pair of $\pi$ pulses on the ${ }^{13} \mathrm{C}$ spin is not enough to keep its state. To show this concept, we will illustrate an extreme case where the pair of $\pi$ pulses applied to the ${ }^{13} \mathrm{C}$ spin does not affect any dynamics on the ${ }^{13} \mathrm{C}$ spin:

$$
\begin{aligned}
\operatorname{Tr}_{\mathrm{e}}( & \left.A d\left(e^{-i 2 H_{\mathrm{se}}\left(\delta_{1}+\delta_{2}\right)}, \rho(t)\right)\right) \\
= & \operatorname{Tr}_{\mathrm{e}}\left(A d \left(e^{-i H_{\mathrm{se}} \delta_{1}}\left({ }^{\mathrm{s}}(\pi)_{\pi} \otimes X\left(\theta_{1}\right)\right) e^{-i H_{\mathrm{se}}\left(\delta_{1}+\delta_{2}\right)}\right.\right. \\
& \left.\left.\quad\left({ }^{\mathrm{s}}(\pi)_{0} \otimes X\left(\theta_{2}\right)\right) e^{-i H_{\mathrm{se}} \delta_{2}}, \rho(t)\right)\right),
\end{aligned}
$$

where $X\left(\theta_{i}\right)$ is an effect of the impurities.

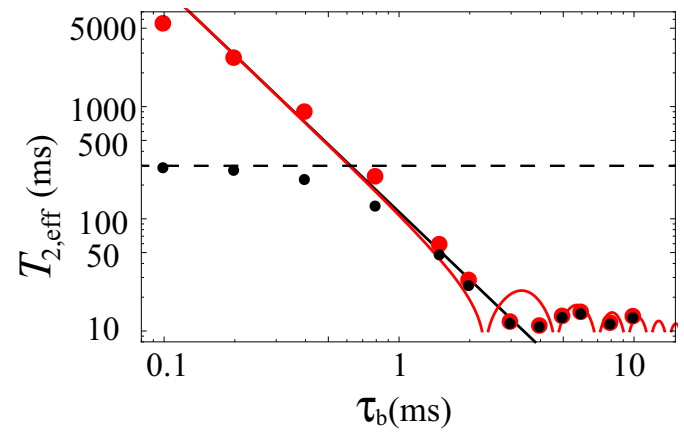

FIG. 3. Effective $T_{2}$ (named $T_{2, \text { eff }}$ ) under DD as a function of the time interval $\tau_{\mathrm{b}}$ between DD pulses ( $\pi$ pulses). The small -'s and big 's are simulation results with and without the direct influence of the magnetic impurities on the ${ }^{13} \mathrm{C}$ spin, respectively. The black line is $f\left(\tau_{\mathrm{b}}\right)=\frac{1.2 \times 10^{-7}}{\tau_{\mathrm{b}}{ }^{2}}$, while the red one is $f\left(\tau_{\mathrm{b}}\right)=$ $\frac{37 \times 10^{-3}}{\log \left(\left|J \tau_{\mathrm{b}} / \sin J \tau_{\mathrm{b}}\right|\right)}$. The dashed line is $T_{2, \text { eff }}=300 \mathrm{~ms}$ and is limited by the direct influence of the magnetic impurities on the ${ }^{13} \mathrm{C}$ spin. See the text for more details.

We simulate DD experiments, where a series of $\pi$ pulses is applied to the ${ }^{13} \mathrm{C}$ spin, by extending the FID simulation based on the noise channel. We assume that the $\pi$ pulses are instantaneously performed without any errors. The DD pulses extend the phase coherence and DD becomes more effective as we decrease the time interval between them, $\tau_{\mathrm{b}}$, as shown in Fig. 2. The extended phase coherence is characterized by an apparent spin-spin relaxation time, named an effective spinspin relaxation time, $T_{2 \text {,eff }}$. We summarize the results of the numerical simulations for DD where we plot $T_{2 \text {,eff }}$ as a function of $\tau_{\mathrm{b}}$ in Fig. 3. We note that there was related theoretical work where the dissipative nature of a subsystem's dynamics was modified by dynamically manipulating the system [53].

We can make an order estimation of the effects of DD as follows. First of all, let us consider the period $\left[0, T_{\mathrm{e}}\right]$ without $\pi$ pulses. Because of magnetic impurities, there must be about one, on average, flip-flop of a ${ }^{1} \mathrm{H}$ spin in $\left[0, T_{\mathrm{e}}\right]$. In this case, the phase of the ${ }^{13} \mathrm{C}$ spin almost completely decays during the period of $T_{\mathrm{e}}$. On the other hand, when a series of $\pi$ pulses with a time interval of $\tau_{\mathrm{b}}$ are applied, the phase change due to the random flip-flop of a ${ }^{1} \mathrm{H}$ spin is described as

$$
\frac{1}{\tau_{\mathrm{b}}} \int_{0}^{\tau_{\mathrm{b}}} e^{-i \frac{J}{2} t} e^{i \frac{J}{2}\left(\tau_{\mathrm{b}}-t\right)} d t=\frac{\sin \frac{J}{2} \tau_{\mathrm{b}}}{\frac{J}{2} \tau_{\mathrm{b}}}
$$

for $J \tau_{\mathrm{b}} \ll 1$, where $t$ denotes the time for the random flip-flop. This phase change is effective only for the time interval $\tau_{\mathrm{b}}$, because we perform multiple $\pi$ pulses for refocusing, as shown in Fig. 4. We can assume that this phase change occurs with a probability of $p=1-e^{-\frac{\tau_{b}}{T_{\mathrm{e}}}} \simeq \frac{\tau_{b}}{T_{\mathrm{e}}}$, while we have no phase decay with a probability of $1-p$. On the other hand, the phase relaxation time constant apparently becomes longer from $T_{\mathrm{e}}$ to

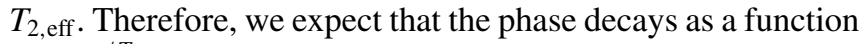
of $e^{-\tau_{b} / T_{2, \text { eff }}}$ during $\tau_{b}$ when the series of $\pi$ pulses is applied. 


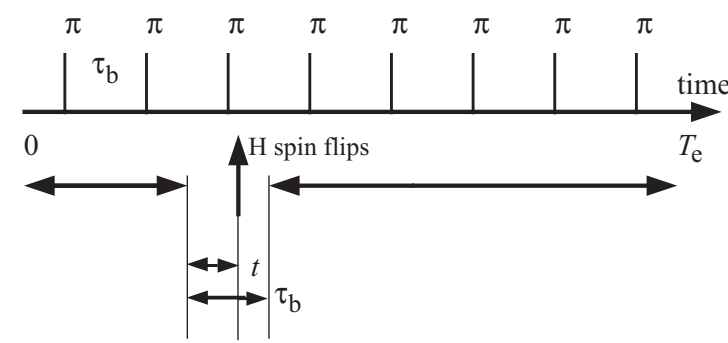

FIG. 4. Schematic of the spin dynamics in our system. The ${ }^{1} \mathrm{H}$ spin randomly flips due to the environment. The up arrow indicates the timing when the ${ }^{1} \mathrm{H}$ spin flips. The effects of $H_{\mathrm{se}}$ during the periods indicated by two direction arrows are canceled due to the $\pi$ pulses.

We obtain

$$
(1-p)+p \frac{\sin \frac{J}{2} \tau_{\mathrm{b}}}{\frac{J}{2} \tau_{\mathrm{b}}} \approx e^{-\tau_{b} / T_{2, \mathrm{eff}}}
$$

or

$$
T_{2, \mathrm{eff}} \approx \frac{24 T_{\mathrm{e}}}{J^{2} \tau_{\mathrm{b}}^{2}} .
$$

We obtain $T_{2, \text { eff }}=1.6 \times 10^{-7} / \tau_{\mathrm{b}}{ }^{2}$ with $T_{\mathrm{e}}=13 \mathrm{~ms}$ and $J=$ $2 \pi \times 215.2 \mathrm{rad} / \mathrm{s}$. On the other hand, we obtain the numerator of $1.2 \times 10^{-7}$ from the simulations, as shown in Fig. 3. Our order estimation predicts the same $\tau_{\mathrm{b}}$ dependence of $T_{2 \text {,eff }}$ as the simulations.

We also evaluate $T_{2, \text { eff }}$ by replacing $\bar{\Delta}$ with $T_{\mathrm{e}}$ in Eq. (60) in our previous publication [36]. We obtain

$$
T_{2, \text { eff }} \approx \frac{T_{\mathrm{e}}}{\ln \left(\left|\frac{J \tau_{b} / 2}{\sin J \tau_{b} / 2}\right|\right)},
$$

which is in reasonable agreement with the simulation results shown in Fig. 3. We obtain the numerator of $37 \mathrm{~ms}$ from the simulations instead of $T_{\mathrm{e}}=13 \mathrm{~ms}$.

We further consider a direct influence of magnetic impurities on a ${ }^{13} \mathrm{C}$ spin. This effect appears to be a longitudinal relaxation of the ${ }^{13} \mathrm{C}$ spin and modifies Eq. (9) as follows:

$$
\begin{aligned}
\rho(t+\delta) \approx & \left(1-\left(\kappa+\kappa^{\prime}\right) \delta\right) A d\left(e^{-i H_{\mathrm{se}} \delta}, \rho(t)\right) \\
& +\kappa \delta A d\left(\left(\sigma_{0} \otimes X(\theta)\right) e^{-i H_{\mathrm{se}} \delta}, \rho(t)\right)+\kappa^{\prime} \delta \rho_{\mathrm{th}},
\end{aligned}
$$

where $\kappa^{\prime}$ is the order of $1 / T_{\mathrm{s}}$, where $T_{\mathrm{s}}$ is the longitudinal relaxation time constant of the ${ }^{13} \mathrm{C}$ spin. Note that the third term $\kappa^{\prime} \delta \rho_{\text {th }}$ has no memory effect (because we do not have a term of $e^{-i H_{\mathrm{se}} \delta}$ ), unlike the second term. Therefore, the third term causes a time-homogeneous behavior of the ${ }^{13} \mathrm{C}$ spin.

By taking into account the direct influence of magnetic impurities on the ${ }^{13} \mathrm{C}$ spin, we simulate DD experiments again. The results are summarized in Fig. 3, too. We take $T_{\mathrm{s}}=1 / \kappa^{\prime}=$ $300 \mathrm{~ms}$ in Fig. 3, which is a measured $T_{1}$ of the ${ }^{13} \mathrm{C}$ spin. These $T_{2, \text { eff }}$ 's are limited by $T_{\mathrm{s}}$, as expected.

\section{Understanding DD as a time domain filter}

The concept of quantum channel provides a convenient way of numerically calculating dynamics of the system under DD, as shown in the previous section [50]. We can also interpret the DD as a time domain filter [12,54]. Decoherence of a system occurs because of noise generated by an environment, which is characterized by the power spectrum density $S(\omega)$. Suppose that the "noise" is periodic of which frequency is $\omega$ and the $\pi$-pulse interval in DD is $\tau_{\mathrm{b}}$. If we measure the "decoherence" caused by the noise for an "infinitely" long period, the "noise" is effective only if $\omega \tau_{\mathrm{b}}=\pi$. Although the noise is neither periodic nor is infinitely long measurement possible, one can imagine that the noise density at $\omega=\frac{\pi}{\tau_{\mathrm{b}}}$ has a large effect under DD.

The noise spectra of environments were measured or estimated by employing DD as a time domain filter $[12,15,55,56]$. It is known that for most of the solid-state systems, the power spectrum density becomes larger as we decrease the frequency. This means that as we decrease the pulse-interval time, the coherence time becomes longer, which has been observed in many systems $[12,15,55,56]$.

\section{DD and time-inhomogeneous noise}

The DD is strongly related to the time inhomogeneity of the noise [57]. When the correlation time of the environment is much smaller than the time resolution of the experimental apparatus, the system shows an exponential decay [51], and this is called a time-homogeneous noise [6-10]. It is known that, for such a time-homogeneous noise, the DD does not improve the coherence time of the system [51]. On the other hand, if we can experimentally observe a time scale much shorter than the environmental correlation time, we could observe a nonexponential decay behavior [11], and such a decoherence is considered as time inhomogeneous. Since the DD is effective when the correlation time of the environment is longer than the time interval of the $\pi$ pulse, the DD is useful to estimate if the noise is time inhomogeneous.

\section{EXPERIMENTS}

We experimentally confirm that a time-inhomogeneous environment is engineered by selecting sample molecules (tuning of a memory effect) and by changing the concentrations of magnetic impurities (tuning of randomness) through DD experiments.

Our NMR spectrometer is a standard one for chemical analysis, or a JEOL ECA-500. We perform several DD experiments with various molecules, such as chloroform $\left(\mathrm{CHCl}_{3}\right)$, carbon tetrachloride $\left(\mathrm{CCl}_{4}\right)$, methanol $\left(\mathrm{CH}_{3} \mathrm{OH}\right)$, trifluoroethanol $\left(\mathrm{CF}_{3} \mathrm{CH}_{2} \mathrm{OH}\right)$, and tetramethylsilane $\left(\mathrm{Si}\left(\mathrm{CH}_{3}\right)_{4}\right)$. In all molecules except for carbon tetrachloride, there is one spin that is surrounded by other spin(s) and that is protected from direct influences of magnetic impurities.

\section{A. Chloroform}

We measure three ${ }^{13} \mathrm{C}$-enriched chloroform samples. They are 66.6-mM, 303-mM, and 181-mM chloroform solutions in acetone-d6, while their magnetic impurity concentrations are $34 \mathrm{mM}, 48 \mathrm{mM}$, and $133 \mathrm{mM}$, respectively, see Table I. It is worth mentioning that if we consider $T_{1}$ or $T_{2}$ multiplied by $\mathrm{Fe}(\mathrm{III})$ concentrations, these quantities are almost constant regardless of the $\mathrm{Fe}(\mathrm{III})$ concentrations. This indicates that chloroform molecules are isolated from each other, and so we only have to consider a single molecule. Although the 
TABLE I. $T_{1}$ and $T_{2}$ of our chloroform samples are summarized. $T_{1}$ and $T_{2}$ of the 200-mM chloroform sample are taken from Ref. [36]. The figures in the parentheses are $T_{1}$ or $T_{2}$ times Fe(III) concentrations.

\begin{tabular}{|c|c|c|c|c|c|c|}
\hline \multicolumn{3}{|c|}{ Sample } & \multicolumn{2}{|c|}{${ }^{1} \mathrm{H}$} & \multicolumn{2}{|c|}{${ }^{13} \mathrm{C}$} \\
\hline No. & $\mathrm{CHCl}_{3}(\mathrm{mM})$ & $\overline{\mathrm{Fe}(\mathrm{III})(\mathrm{mM})}$ & $T_{1}(\mathrm{~ms})$ & $T_{2}(\mathrm{~ms})$ & $T_{1}(\mathrm{~s})$ & $T_{2}(\mathrm{~ms})$ \\
\hline I & 303 & 48 & $6.5\left(3.1 \times 10^{2}\right)$ & $7.0\left(3.4 \times 10^{2}\right)$ & $0.30\left(1.4 \times 10^{1}\right)$ & $17\left(8.2 \times 10^{2}\right)$ \\
\hline II & 66.6 & 34 & $9.1\left(3.1 \times 10^{2}\right)$ & $8.7\left(3.0 \times 10^{2}\right)$ & $0.43\left(1.5 \times 10^{1}\right)$ & $20\left(9.6 \times 10^{2}\right)$ \\
\hline \multirow[t]{2}{*}{ III } & 181 & 133 & $2.2\left(2.9 \times 10^{2}\right)$ & $2.1\left(2.9 \times 10^{2}\right)$ & $0.10\left(1.3 \times 10^{1}\right)$ & $5\left(6.7 \times 10^{2}\right)$ \\
\hline & 200 & 0 & $20 \mathrm{~s}$ & $7.5 \mathrm{~s}$ & $20 \mathrm{~s}$ & $0.3 \mathrm{~s}$ \\
\hline
\end{tabular}

${ }^{13} \mathrm{C}$ spins' $T_{2}$ multiplied by Fe(III) concentrations show some variations compared with the others, this can be explained by the fact that they are influenced by not only the magnetic impurities but also the $J$ coupling.

Our simulations discussed in Sec. II are based on the parameters measured with sample I. The measured $T_{1}$ and $T_{2}$ of a ${ }^{1} \mathrm{H}$ spin are both about $7 \mathrm{~ms}$. It is reasonable because magnetic impurities simultaneously flip the ${ }^{1} \mathrm{H}$ spins and destroy their phase coherence. On the other hand, a measured $T_{1}$ of a ${ }^{13} \mathrm{C}$ spin is $300 \mathrm{~ms}$, while its $T_{2}$ is $17 \mathrm{~ms}$. Without magnetic impurities, $T_{1}$ and $T_{2}$ of a ${ }^{13} \mathrm{C}$ spin are $20 \mathrm{~s}$ and $0.3 \mathrm{~s}$, respectively [36]. The spin-spin $\left(T_{2}\right)$ relaxation is increased by about 20 times via the addition of magnetic impurities. We measured the $T_{1}$ using the standard inversion recovery sequence [45]. Due to a good field homogeneity, $T_{2}$ can be obtained directly from a FID signal [45], see Fig. 2. For our sample, $J / 2 \pi=215.2 \mathrm{~Hz}$.

The measured FID signal is well reproduced with the model described in Sec. II B 3, as shown in Fig. 2 [50]. The only fitting parameter is $\kappa$, and we take $1 / \kappa=13 \mathrm{~ms}$. This is about twice the of the measured $T_{2}(7 \mathrm{~ms})$ of a ${ }^{1} \mathrm{H}$ spin, as we mentioned before.

We perform DD experiments with our three samples. The

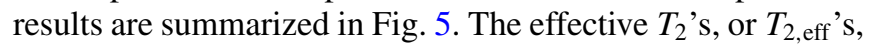

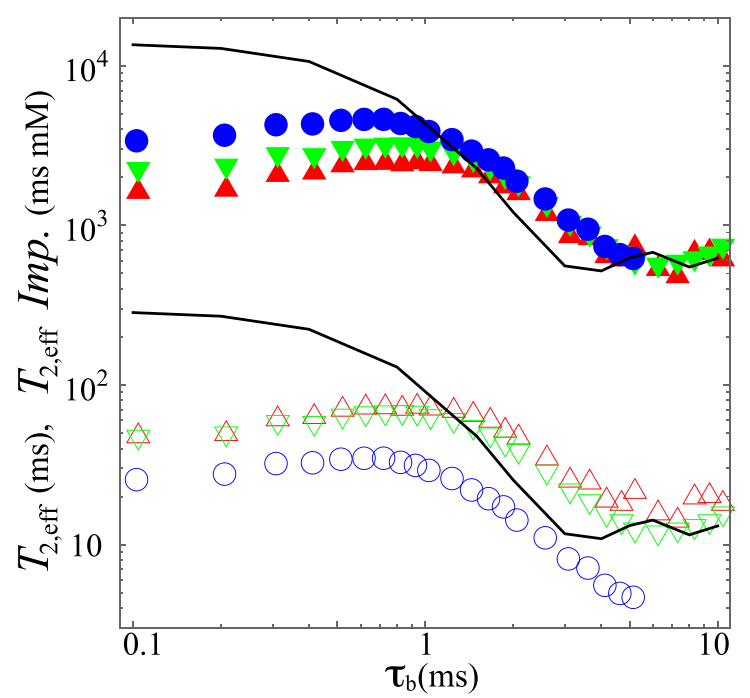

FIG. 5. Effective $T_{2}$ 's ( $T_{2, \text { eff' }}$ 's) of the chloroform samples under DD. The data of samples I $(\nabla)$, II $(\triangle)$, and III (O) are shown. The solid line is the simulated $\tau_{\mathrm{b}}$ dependence of $T_{2 \text {,eff }}$ shown in Fig. 3. The filled symbols are $\left(T_{2, \text { eff }} \times\right.$ Impurity concentration). of ${ }^{13} \mathrm{C}$ are measured through the application of XY-8 sequences [58,59], which compensates pulse imperfections, to the ${ }^{13} \mathrm{C}$ spin.

We observe the following at $\tau_{\mathrm{b}}>1 \mathrm{~ms}$ :

(a) $T_{2, \text { eff }}$ 's become larger as we decrease $\tau_{\mathrm{b}}$ 's.

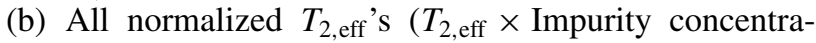
tion) fall onto the universal curve regardless of the magnetic impurity concentrations.

(c) This universal curve is well approximated with the simulation results when the direct influence of the magnetic impurities is taken into account, see Fig. 3.

These indicate that DD works well when $\tau_{\mathrm{b}} \geqslant 1 \mathrm{~ms}$. We found the following, however, at $\tau_{\mathrm{b}}<1 \mathrm{~ms}$ :

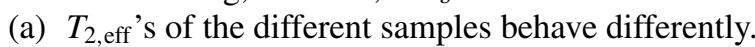

(b) The signals under DD often decay nonexponentially. Note that $T_{2, \text { eff's }}$, calculated by fitting the data with a single exponential function, are plotted in Fig. 5.

These observations imply that DD does not work well when $\tau_{\mathrm{b}} \leqslant 1 \mathrm{~ms}$. We discuss some possible origins of this malfunction in Sec. IV.

\section{B. Carbon tetrachloride}

We measure the carbon tetrachloride sample in order to compare with the chloroform samples. The molecular structure is shown as an inset of Fig. 6. This sample is not ${ }^{13} \mathrm{C}$ enriched

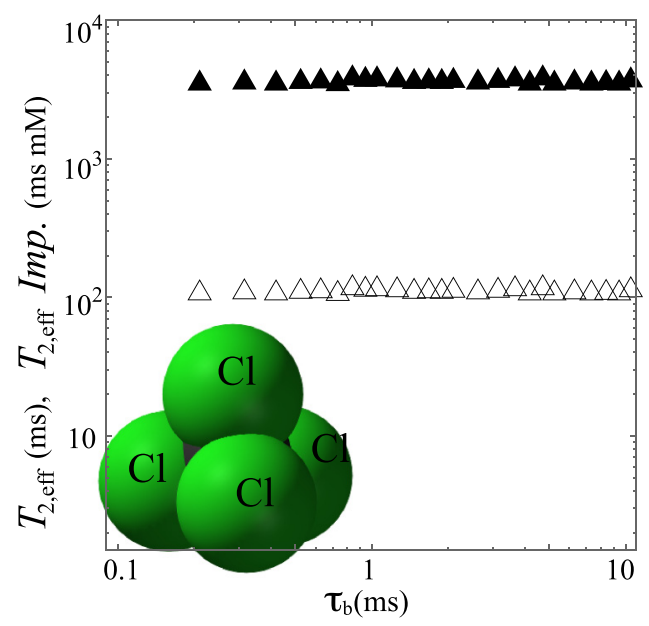

FIG. 6. Effective $T_{2}$ 's ( $T_{2, \text { eff }}$ 's) of the carbon tetrachloride sample under DD. The Fe(III)acac concentration is $33 \mathrm{mM}$. The filled symbols are $\left(T_{2, \text { eff }} \times\right.$ Impurity concentration) in order to compare these data with those in Fig. 5. The inset shows a molecular structure of carbon tetrachloride. 


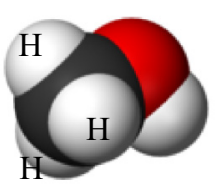

(a)

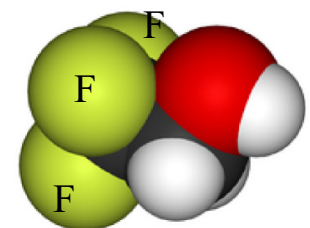

(b)
FIG. 7. Molecular structures of (a) methanol and (b) trifluoroethanol. Our trifluoroethanol sample is not ${ }^{13} \mathrm{C}$ enriched and thus the environment of the ${ }^{13} \mathrm{C}$ spin in $\mathrm{CF}_{3}$ is only the surrounding three ${ }^{19} \mathrm{~F}$ spins.

and is a mixture of carbon tetrachloride and acetone-d6, of which weight ratio is $8.7: 1$. The magnetic impurity of $\mathrm{Fe}(\mathrm{III})$ acac of $33 \mathrm{mM}$ is added.

We expect that there are only direct influences of magnetic impurities on a ${ }^{13} \mathrm{C}$ spin in the carbon tetrachloride sample, since surrounding $\mathrm{Cl}$ spins are magnetically inert in the time scale of NMR. In other words, $H_{\mathrm{se}}=0$.

Actually, we obtain $T_{2 \text {,eff }}$ that is independent of $\tau_{\mathrm{b}}$, as shown in Fig. 6. The strength $\left(\sim 1 / 100 \mathrm{~ms}^{-1}\right)$ of this direct influence is the same order as that $\left(\sim 1 / 300 \mathrm{~ms}^{-1}\right)$ of chloroform [34 mM of $\mathrm{Fe}(\mathrm{acac})]$, as we expected. We conclude that the dephasing in this sample is time homogeneous where the memory effect of the environment is negligible.

\section{Methanol and trifluoroethanol}

We measure the methanol and trifluoroethanol samples in order to clarify the role of the scalar coupling strength between a system and its engineered environment, or the time scale of the memory effect for the time inhomogeneity. We focus on the relaxation of the ${ }^{13} \mathrm{C}$ spin in a $\mathrm{CH}_{3}$ group of methanol and $\mathrm{CF}_{3}$ group in trifluoroethanol. Trifluoroethanol is not ${ }^{13} \mathrm{C}$ enriched and thus the engineered environment of the ${ }^{13} \mathrm{C}$ spin in the $\mathrm{CF}_{3}$ group is approximately the only surrounding three ${ }^{19} \mathrm{~F}$ spins. The environment of the ${ }^{13} \mathrm{C}$ spin in the $\mathrm{CH}_{3}$ group is also the three ${ }^{1} \mathrm{H}$ spins, see Fig. 7. The difference between these two engineered environments for the ${ }^{13} \mathrm{C}$ spin is a strength of the scalar coupling constant. They are $2 \pi \times 140 \mathrm{rad} / \mathrm{s}$ and $2 \pi \times 277 \mathrm{rad} / \mathrm{s}$ for $\mathrm{CH}_{3}$ and $\mathrm{CF}_{3}$, respectively. These different $J$ 's are caused by the different electronegativity of $\mathrm{H}$ and $\mathrm{F}$ atoms.

The methanol sample is $1.4 \mathrm{M}{ }^{13} \mathrm{C}$ enriched methanol solved in acetone-d6. We prepare four samples of which the magnetic impurity [Fe(III) acetylacetonate] concentrations are $12 \mathrm{mM}, 21 \mathrm{mM}, 49 \mathrm{mM}$, and $79 \mathrm{mM}$. The trifluoroethanol samples are mixture of trifluoroethanol (not ${ }^{13} \mathrm{C}$ enriched) and acetone-d6 of which the weight ratio is 5.4:1. We prepare four samples having magnetic impurity concentrations of $3.8 \mathrm{mM}$, $12 \mathrm{mM}, 27 \mathrm{mM}$, and $48 \mathrm{mM}$. We, however, do not show $T_{2 \text {, eff's }}$ of the 3.8-mM and 12-mM magnetic impurity trifluoroethanol samples because these magnetic impurities are too small to be a dominant relaxation source for the ${ }^{13} \mathrm{C}$ spin in the $\mathrm{CF}_{3}$ group.

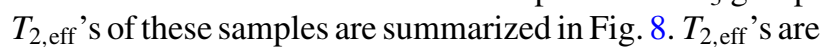
first multiplied by the impurity concentrations. These data of the methanol samples fall onto one universal curve, while those of the trifluoroethanol samples fall on another universal curve. Then we normalize these two curves so that the normalized

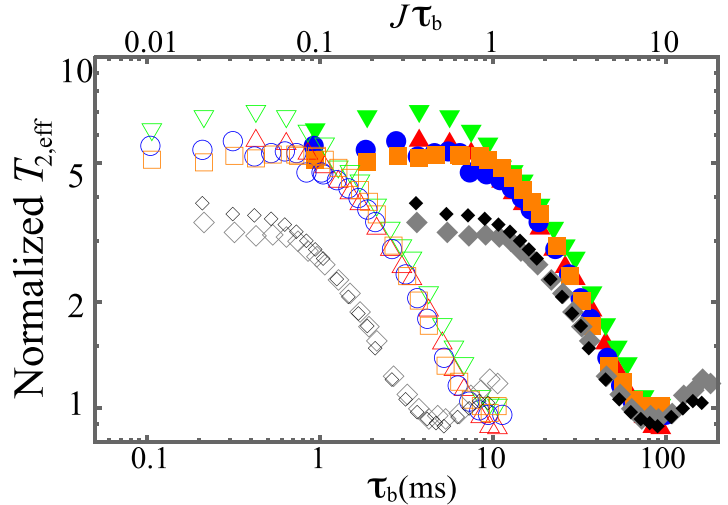

FIG. 8. Normalized $T_{2, \text { eff }}$ 's of the methanol and trifluoroethanol samples under DD. The Fe(III)acac concentrations of the methanol samples are $12 \mathrm{mM}(\triangle), 21 \mathrm{mM}(\nabla), 49 \mathrm{mM}(\mathrm{O})$, and $79 \mathrm{mM}(\square)$, while those of the trifluoroethanol samples are $27 \mathrm{mM}(\checkmark)$ and $48 \mathrm{mM}$ $(\diamond)$. The filled symbols are the normalized $T_{2, \text { eff }}$ 's but as a function of $J \tau_{\mathrm{b}}$ (the top horizontal axis).

$T_{2, \text { eff }}$ 's can be unity for large $\tau_{\mathrm{b}}$ 's. The open symbols in Fig. 8 show these normalized $T_{2 \text {,eff }}$ as a function of $\tau_{\mathrm{b}}$. On the other hand, the filled symbols are these normalized $T_{2 \text {,eff }}$ as a function of $J \tau_{\mathrm{b}} . T_{2, \text { eff }}$ 's start to increase at $J \tau_{\mathrm{b}} \approx 10$ for all samples, and thus we conclude that the time scale of the ${ }^{13} \mathrm{C}$ spin dynamics is determined by the scalar coupling constant $J$, as we expected. The behavior at $J \tau_{\mathrm{b}}<1$ is influenced by the direct interaction of the ${ }^{13} \mathrm{C}$ spin with magnetic impurities such as in the case of chloroform, and thus these normalized $T_{2, \text { eff's are apart from the universal curve. }}$

\section{Tetramethylsilane}

We now discuss the results with a tetramethylsilane (TMS) molecule of which the FID signal shows revivals, as shown in Fig. 9. We interpret this as a non-Markovian effect [5], where the information flows back from the environment to our system. On the other hand, the sample with $40 \mathrm{mM}$ of magnetic impurity shows a monotonous decay as shown in the inset of Fig. 9. Here, the information of the system

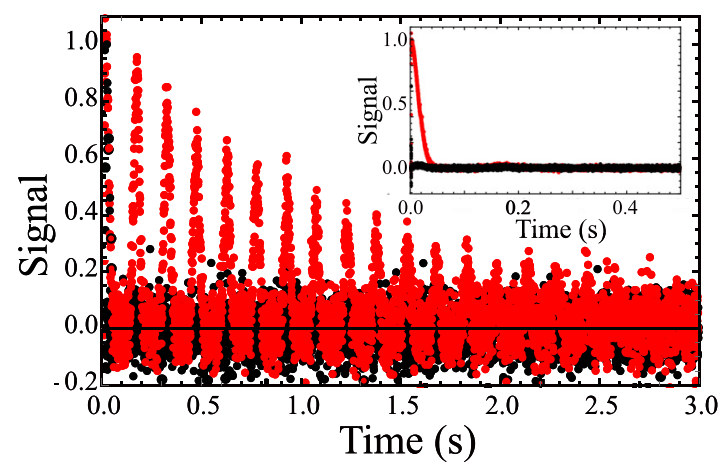

FIG. 9. FID signal of tetramethylsilane sample without magnetic impurities, while the inset shows the FID signal with $40 \mathrm{mM}$ of magnetic impurity. The red (black) points are real (imaginary) parts of the signal. The difference in noise of these FID signals is caused by different averaging and thus it is not essential. 


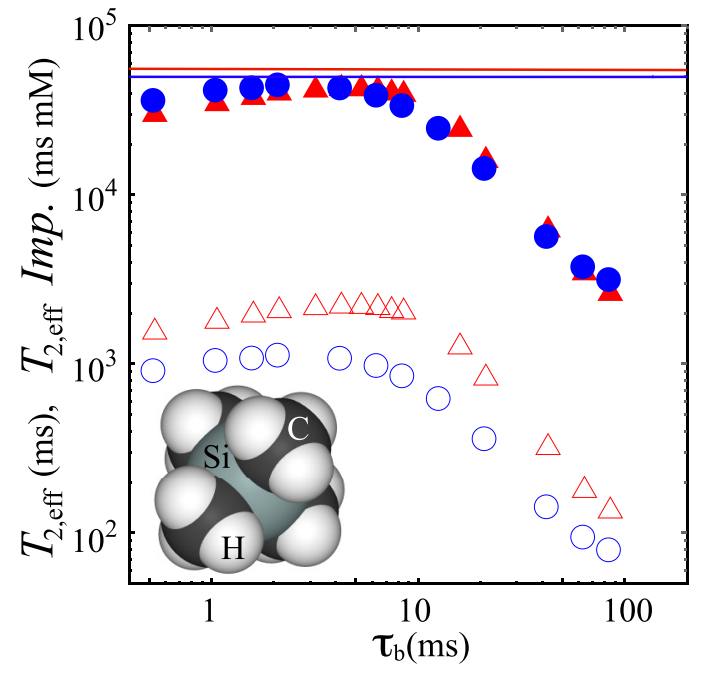

FIG. 10. Effective $T_{2}^{\prime} s$ ( $T_{2, \text { eff }}$ ) of TMS under DD. Fe(III)acac concentrations are $(\triangle) 19 \mathrm{mM}$ and $(O) 40 \mathrm{mM}$. Filled symbols are ( $T_{2 \text {,eff }} \times$ Impurity concentration) in order to normalize the effects of magnetic impurities. The red (blue) solid line is $T_{1} \times$ magnetic impurity.

monotonically leaks into the environment without any revivals. These measurements may show our ability to control the non-Markovianity of the sample, although further research is needed to assess whether these dynamics can be characterized by a measure described in [5]. These will be discussed in more detail in a forthcoming paper.

Note also that a $\mathrm{Si}$ spin (our system) in the center of the molecule is surrounded by four $-\mathrm{CH}_{3}$ groups and is protected from the environment [60]. Therefore this molecule is also suitable for our experiment. Our sample is not ${ }^{13} \mathrm{C}$ enriched and thus we can ignore the $\mathrm{C}$ spins.

Our tetramethylsilane sample is a mixture of $2.89 \mathrm{~g}$ normal (neither ${ }^{13} \mathrm{C}$ nor ${ }^{29} \mathrm{Si}$ is enriched) tetramethylsilane and $2.53 \mathrm{~g}$ acetone-d6. We prepare three samples with magnetic impurities of $0 \mathrm{mM}, 19 \mathrm{mM}$, and $40 \mathrm{mM}$. Their $T_{1}$ 's of Si are $16 \mathrm{~s}(0 \mathrm{mM}), 2.6 \mathrm{~s}(19 \mathrm{mM})$, and $1.4 \mathrm{~s}(40 \mathrm{mM})$.

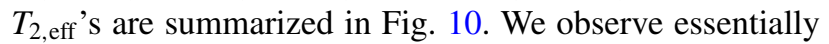
the same behaviors of $T_{2 \text {,eff }}$ vs $\tau_{\mathrm{b}}$ as those of chloroform, in spite of their very small $J$ values of $J /(2 \pi) \approx 6.6 \mathrm{~Hz}$. For example, $T_{2, \text { eff }}$ 's are limited by $T_{1}$ 's, as shown in Fig. 10.

\section{DISCUSSIONS}

The model discussed in Sec. II B qualitatively explains all our experiments, from the FID measurements to DD experiments. There are, however, subtle differences between predictions by the model and our experimental observations. For example:

(1) $T_{2, \text { eff }} \times$ magnetic impurity concentrations at small $\tau_{\mathrm{b}}$ do not fall onto universal curves.

(2) The largest $T_{2, \text { eff }}$ 's are less than $T_{1}$.

(3) The decay of the signal is nonexponential when $\tau_{b}$ 's are small.

We attribute these discrepancies not to the imperfect model but to imperfect DD pulses, as pointed out in [12,19,25,61] according to the following discussions, although we employ

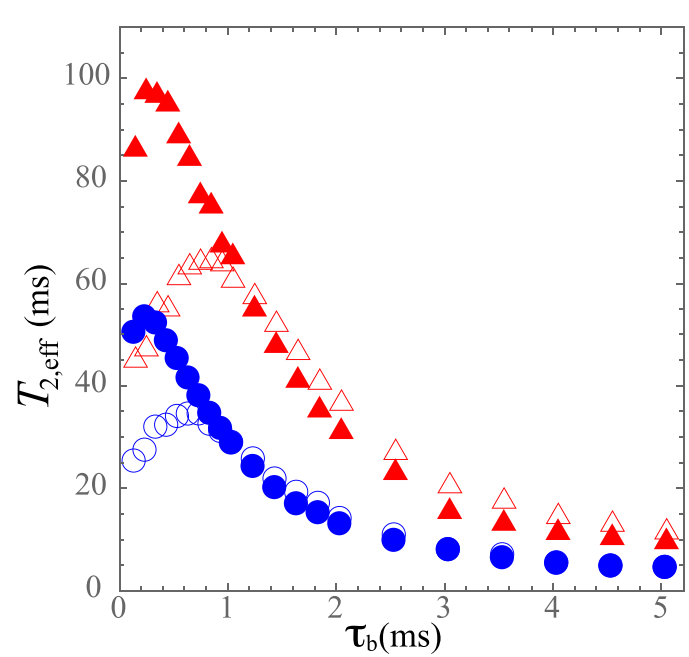

FIG. 11. $T_{2, \text { eff }}$ 's when series of $\pi$ pulses are applied to ${ }^{13} \mathrm{C}$ (open symbols) and ${ }^{1} \mathrm{H}$ (filled ones) spin of the chloroform samples: $(\triangle$, $\Delta)$ sample I and $(\mathrm{O}, \bullet)$ sample III.

XY-8 pulses $[12,58,59]$ that compensate some imperfections of them.

We measure $T_{2, \text { eff }}$ 's of the chloroform samples by applying a series of $\pi$ pulses to a ${ }^{1} \mathrm{H}$ spin, which will provide us with more information about this system. This is one of the benefits of our model: The environment is well defined and its part can be controlled. The experimental results are summarized in Fig. 11. We find the following:

(1) At $\tau_{\mathrm{b}}>1 \mathrm{~ms}, T_{2, \text { eff }}$ 's measured by applying DD pulses ( $\pi$ pulses) to a ${ }^{1} \mathrm{H}$ spin are almost the same as those to ${ }^{13} \mathrm{C}$ spin.

(2) At $\tau_{\mathrm{b}}<1 \mathrm{~ms}$, the DD pulses to $\mathrm{a}{ }^{13} \mathrm{C}$ spin are less effective than those on a ${ }^{1} \mathrm{H}$ spin.

Due to the symmetry of $H_{\text {se }}$ between two spins, DD on the ${ }^{1} \mathrm{H}$ spin should be equivalent to that on the ${ }^{13} \mathrm{C}$ spin if the noise source is only magnetic impurities. As one can see from Fig. 11, this is not the case. The differences between $T_{2 \text {,eff' }}$ ' under DD on the ${ }^{13} \mathrm{C}$ and ${ }^{1} \mathrm{H}$ spins immediately indicate that the DD operation itself acts as some other decoherence source of the ${ }^{13} \mathrm{C}$ spin, except for the magnetic impurities, because the directly applied $\pi$ pulses on ${ }^{13} \mathrm{C}$ spins are more error-sensitive than those on ${ }^{1} \mathrm{H}$ ones. We should also remember that we engineered the environment and controlled the environmental noise. This is guaranteed from the much shorter $T_{1}$ 's and $T_{2}$ 's compared with those of the sample without magnetic impurities, shown in Table I. Therefore, we can be sure that the noise from the environment cannot generate the maxima in Fig. 11.

In order to confirm that the pulse imperfections cause the maxima in Fig. 11, we investigate relaxations at $\tau_{\mathrm{b}} \lesssim 1 \mathrm{~ms}$ in more detail. Figure 12 shows the relaxations of sample I of chloroform at $\tau_{\mathrm{b}}=0.1,0,2,0.4,0.8,1.2$, and $1.6 \mathrm{~ms}$ with different $\pi$-pulse amplitudes. We notice the following:

(1) The smaller $\tau_{\mathrm{b}}$ leads to larger nonexponentiality in the decay.

(2) The pulse amplitude errors lead to the initial nonexponential decay but they do not have a large influence on the long-term decay behavior. 


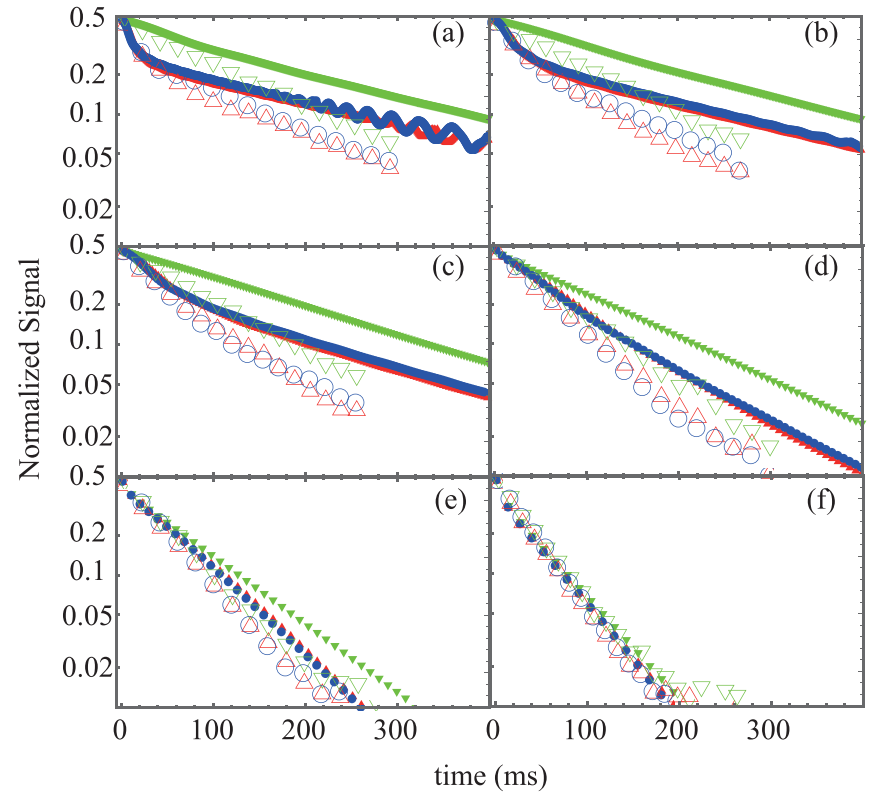

FIG. 12. Signal decays of sample I (chloroform) under DD. Panels (a)-(f) show the signal decays when $\tau_{\mathrm{b}}=0.1,0.2,0.4,0.8,1.2$, and $1.6 \mathrm{~ms}$, respectively. The large open $\nabla$ 's are measured when refocusing $(\pi)$ pulses are optimal, while the large open $\triangle$ 's (O's) are done with pulses of which rotation angles are 0.9 (1.1) times smaller (larger) than its optimal value. The small filled symbols are simulation results in which the pulse strength distribution in the sample is considered. The small filled $\nabla$ 's are simulation results when refocusing $(\pi)$ pulses are optimal, while the small filled $\Delta$ 's $(\bullet$ 's) are those with pulses of which rotation angles are 0.9 (1.1) times smaller (larger) than its optimal value.

(3) The effect of pulse amplitude errors becomes smaller when $\tau_{\mathrm{b}}$ is larger and seems to be negligible when $\tau_{\mathrm{b}} \geqslant 1.6 \mathrm{~ms}$.

A similar nonexponential decay like in Fig. 12 under DD was observed and attributed to pulse imperfections in DD $[12,15,19,25,59,61]$. Then we try to reproduce these observations by assuming imperfections in DD pulses that are not considered in Sec. II. First, we take into account the distribution of rf pulse strengths in the sample according to our previous publication [62] and the finite $\pi-$ pulse length of $\sim 100 \mu \mathrm{s}$. The simulation results are summarized and compared with experimental results in Fig. 12. It seems that the origin of the initial nonexponential decay is caused by the distribution of the pulse strength. This, however, cannot explain the long-term faster relaxation than that predicted by the simulations.

Second, we introduce fluctuations of the pulse amplitude and errors in the pulse phase in our simulations, see Fig. 13. We here assume that the pulse amplitude changes $5 \%$ of that of the $\pi$ pulse randomly with a rectangular distribution. We also assume that the phase has an error of $0.1 \mathrm{rad}$ and fluctuates $0.1 \mathrm{rad}$ randomly. From these analysis, we have shown that the faster decay in the longer term can qualitatively be reproduced by the fluctuations of the $\pi$-pulse parameters [19], at least if we choose the parameters mentioned above.

Our experimental conditions at $\tau_{\mathrm{b}} \lesssim 1 \mathrm{~ms}$ are far beyond expectations by the manufacturer of our NMR spectrometer, and thus these pulse imperfections might occur in our experi-

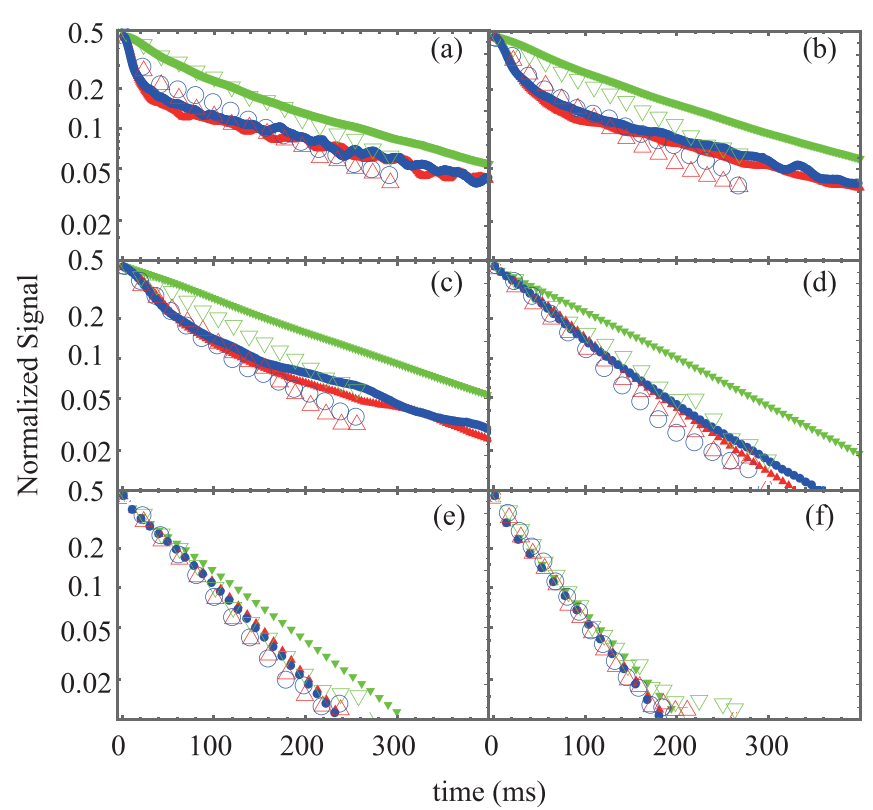

FIG. 13. Simulated signal decays of sample I (chloroform), when pulse amplitude and phase fluctuations exist, are shown. Panels (a)-(f) are results when $\tau_{\mathrm{b}}=0.1,0.2,0.4,0.8,1.2$, and $1.6 \mathrm{~ms}$, respectively. For comparison, corresponding measured signal decays of sample I are shown, too. The small $\nabla$ 's are simulation results when $\pi$ pulses are optimal, while the $\Delta$ 's $(\bullet$ 's) are those with pulses of which rotation angles are 0.9 (1.1) times $\pi$. The open large symbols are experimental results (already shown in Fig. 12).

ments and explain the nonideal behaviors of DD experiments when $\tau_{\mathrm{b}}$ 's are small.

\section{DISCUSSION AND CONCLUSION}

We remark on the prospects for the demonstration of our ideas with the other systems. The basis of our idea to control the decoherence is the use a coupled two-qubit system in NMR, where one of the qubits has a longer coherence time than the other. By treating the short-lived qubit as a part of the environment for the long-lived system, we can control the environment. Also, we can decrease the coherence time of the short-lived system by adding magnetic impurities. Note that although adding magnetic impurities into the sample to reduce $T_{1}$ and $T_{2}$ is a known technique in NMR [44], it is not common to reduce $T_{2}$ intentionally, because a short $T_{2}$ has very little or no merit for usual NMR measurements. On the other hand, we use this technique to control time-inhomogeneous dephasing noise sources [6-10,43], which plays an important role in quantum information processing. The time-inhomogeneous decay process is characterized by a time-dependent decoherence rate that leads to a nonexponential decay, while time-homogeneous decay shows an exponential decay with a constant decoherence rate $[6,8,11]$. Such a time-inhomogeneous noise provides a significant difference from the time-homogeneous noise when we employ a quantum system for QIP. For example, although the dynamical decoupling (DD) is one of the typical decoherence suppression techniques for QIP [12], DD is effective only when the noise is time inhomogeneous. Moreover, in quantum metrology, entanglement sensors can show 
quantum advantages over classical sensors when the decay process is time inhomogeneous $[6,63,64]$, while entanglement sensors have the same performance as classical sensors with exponential decay processes [65]. The quantum Zeno effect is another example where time-inhomogeneous noise becomes important. The quantum Zeno effect does not appear if the decoherence shows an exponential decay [21], while we can utilize the quantum Zeno effect if the environment has the time-inhomogeneous properties [23,50]. Our model actually provides an efficient way to emulate the time-inhomogeneous decoherence by engineering the environment itself.

Although we choose to demonstrate our decoherence model where a short-lived system is coupled with a long-lived system by the NMR due to its simplicity, it is also possible to apply our idea to another system. In other words, our model can be regarded as an simulator of more practical systems for quantum information processing (QIP) and can also be employed for evaluating various decoherence suppression techniques. For example, a superconducting qubit coupled with a quantum memory such as a microwave resonator, a spin ensemble, or microscopic two-level system is one such candidate [66-68]. A nitrogen-vacancy center in diamond is also another one where an electron spin is coupled with a long-lived nuclear spin via a hyperfine coupling [69]. Here we propose a convenient test bench in order to examine decoherence suppression techniques for various important systems. Moreover, our model is not necessarily limited to simulating systems for QIP but may also be employed for studying (simulating) some other interesting phenomena. A decoherence-induced dynamical phase transition [70] could be such an example because of the similarity of the models. Our model should also be useful to study time-inhomogeneous noise because its memory effect and randomness are independently controlled.
In conclusion, we have proposed a NMR model that is useful for both understanding decoherence and evaluating decoherence suppression techniques. By adding magnetic impurities during the sample preparation, we can engineer a real environment to interact with the system that contains the information, while most of the previous works focus on a simulated environment that experimentalists control via active operations such as pulse implementation. Importantly, our model is well described by a quantum channel theory. In addition to the simplicity of the sample preparation, the measurements can be easily performed with the standard high-precision NMR spectrometer that is widely used for chemical analysis in the field of drug discovery. These properties are prerequisite for a useful model, and therefore our model is useful for many experimentalists who hope to investigate decoherence dynamics and to evaluate related techniques.

We have already employed this model in order to perform a principle-of-proof experiment of decoherence suppression by the quantum Zeno effect [50] and have presented the evaluations of dynamical decoupling in this work. We are planning to study the quantum limit of magnetic field sensing [63] with this model.

\section{ACKNOWLEDGMENTS}

We would like to thank Masamitsu Bando, Jefferson G. Filgueiras, and Mikio Nakahara for participating in fruitful discussions. Y.K. would like to thank Grants-in-Aid for Scientific Research from JSPS (Grant No. 25400422) for partial support. This work was also partially supported by JSPS KAKENHI Grant No. 15K17732 and by MEXT KAKENHI Grant No. $15 \mathrm{H} 05870$.
[1] M. A. Nielsen and I. L. Chuang, Quantum Computation and Quantum Information (Cambridge University Press, Cambridge, UK, 2000).

[2] K.-E. Hellwig and K. Kraus, Commun. Math. Phys. 11, 214 (1969).

[3] E. C. G. Sudarshan, P. M. Mathews, and J. Rau, Phys. Rev. 121, 920 (1961).

[4] S. Llyod, Science 273, 1073 (1996).

[5] H. Breuer, E. Laine, J. Piilo, and B. Vacchini, Rev. Mod. Phys. 88, 021002 (2016).

[6] A. Smirne, J. Kołodyński, S. F. Huelga, and R. DemkowiczDobrzański, Phys. Rev. Lett. 116, 120801 (2016).

[7] R. Nichols, T. R. Bromley, L. A. Correa, and G. Adesso, Phys. Rev. A 94, 042101 (2016).

[8] A. Rivas, S. F. Huelga, and M. B. Plenio, Rep. Prog. Phys. 77, 094001 (2014)

[9] A. Rivas, S. F. Huelga, and M. B. Plenio, Phys. Rev. Lett. 105, 050403 (2010).

[10] G. Schaller and T. Brandes, Phys. Rev. A 78, 022106 (2008).

[11] H. Nakazato, M. Namiki, and S. Pascazio, Int. J. Mod. Phys. B 10, 247 (1996).

[12] D. Suter and G. A. Álvarez, Rev. Mod. Phys. 88, 041001 (2016).
[13] D. Bacon, in Quantum Error Correction, edited by D. A. Lidar and T. A. Brun (Cambridge University Press, New York, 2013), and references therein.

[14] L. Viola, Experimental Dynamical Decoupling in Quantum Error Correction, edited by D. A. Lidar and T. A. Brun (Cambridge University Press, New York, 2013), and references therein.

[15] G. De Lange, Z. H. Wang, D. Riste, V. V. Dobrovitski, and R. Hanson, Science 330, 60 (2010).

[16] G. M. Palma, K. A. Suominen, and A. K. Ekert, Proc. R. Soc. London, Ser. A 452, 567 (1996).

[17] W. Yang, Z. Wang, and R. Liu, Front. Phys. 6, 2 (2011).

[18] W. M. Witzel and S. Das Sarma, Phys. Rev. B 74, 035322 (2006); Phys. Rev. Lett. 98, 077601 (2007); W. Yao, R. B. Liu, and L. J. Sham, Phys. Rev. B 74, 195301 (2006); S. K. Saikin, W. Yao, and L. J. Sham, ibid. 75, 125314 (2007); W. Yang and R. B. Liu, ibid. 78, 085315 (2008); 79, 115320 (2009).

[19] H. Gutmann, F. K. Wilhelm, W. M. Kaminsky, and S. Lloyd, Bang-Bang Refocusing of a Qubit Exposed to Telegraph Noise, in Experimental Aspects of Quantum Computing, edited by H. O. Everitt (Springer, New York, 2005), and references therein.

[20] See, also, references cited in [17]. 
[21] B. Mishra and E. C. G. Sudarshan, J. Math. Phys. 18, 756 (1977).

[22] Quantum Error Correction, edited by D. A. Lidar and T. A. Brun (Cambridge University Press, Cambridge, UK, 2013), and references therein.

[23] Y. Matsuzaki, S. Saito, K. Kakuyanagi, and K. Semba, Phys. Rev. B 82, 180518 (2010).

[24] See, for example, a review paper by A. M. Souza, G. A. Álvarez, and D. Suter, Philos. Trans. R. Soc., A 370, 4748 (2012), and references therein.

[25] G. A. Álvarez, A. M. Souza, and D. Suter, Phys. Rev. A 85, 052324 (2012).

[26] G. Balasubramanian, P. Neumann, D. Twitchen, M. Markham, R. Kolesov, N. Mizuochi, J. Isoya, J. Achard, J. Beck, J. Tissler, V. Jacques, P. R. Hemmer, F. Jelezko, and J. Wrachtrup, Nat. Mater. 8, 383 (2009).

[27] Y. Kondo, J. Phys. Soc. Jpn. 76, 104004 (2007).

[28] W. H. Zurek, Rev. Mod. Phys. 75, 715 (2003).

[29] D. G. Cory, M. D. Price, W. Maas, E. Knill, R. Laflamme, W. H. Zurek, T. F. Havel, and S. S. Somaroo, Phys. Rev. Lett. 81, 2152 (1998).

[30] E. M. Fortunato, L. Viola, J. Hodges, G. Teklemariam, and D. G. Cory, New J. Phys. 4, 5 (2002).

[31] L. Xiao and J. Jones, Phys. Lett. A 359, 424 (2006).

[32] T. Kohmoto, Y. Fukuda, M. Kunitomo, K. Ishikawa, Y. Takahashi, K. Ebina, and M. Kaburagi, Phys. Rev. B 52, 13475 (1995).

[33] A. Soare, H. Ball, D. Hayes, X. Zhen, M. C. Jarratt, J. Sastrawan, H. Uys, and M. J. Biercuk, Phys. Rev. A 89, 042329 (2014).

[34] M. J. Biercuk, H. Uys, A. P. VanDevender, N. Shiga, W. M. Itano, and J. J. Bollinger, Nature (London) 458, 996 (2009).

[35] G. Teklemariam, E. M. Fortunato, C. C. López, J. Emerson, J. P. Paz, T. F. Havel, and D. G. Cory, Phys. Rev. A 67, 062316 (2003).

[36] Y. Kondo, M. Nakahara, S. Tanimura, S. Kitajima, C. Uchiyama, and F. Shibata, J. Phys. Soc. Jpn. 76, 074002 (2007).

[37] S. S. Hegde and T. S. Mahesh, Phys. Rev. A 89, 062317 (2014).

[38] J. Morton, A. Tyryshkin, A. Ardavan, S. Benjamin, K. Porfyrakis, S. Lyon, and G. Briggs, Nat. Phys. 2, 40 (2006).

[39] J. Chiaverini, D. Leibfried, T. Schaetz, M. D. Barrett, R. B. Blakestad, J. Britton, W. M. Itano, J. D. Jost, E. Knill, C. Langer, R. Ozeri, and D. J. Wineland, Nature (London) 432, 602 (2004).

[40] Y. Kondo, C. Bagnasco, and M. Nakahara, Phys. Rev. A 88, 022314 (2013).

[41] E. W. Streed, J. Mun, M. Boyd, G. K. Campbell, P. Medley, W. Ketterle, and D. E. Pritchard, Phys. Rev. Lett. 97, 260402 (2006); J. Bernu, S. Deléglise, C. Sayrin, S. Kuhr, I. Dotsenko, M. Brune, J. M. Raimond, and S. Haroche, ibid. 101, 180402 (2008).

[42] L. Viola, E. M. Fortunato, S. Lloyd, C.-H. Tseng, and D. G. Cory, Phys. Rev. Lett. 84, 5466 (2000).

[43] N. Boulant, M. A. Pravia, E. M. Fortunato, T. F. Havel, and D. G. Cory, Quantum Inf. Process. 1, 135 (2002).

[44] T. D. W. Claridge, High-Resolution NMR Techniques in Organic Chemistry (Elsevier Science, Amsterdam, 1999).

[45] M. H. Levitt, Spin Dynamics (John Wiley \& Sons, New York, 2001).

[46] A. Papoulis, Probability, Random Variables and Stochastic Processes (McGraw-Hill, New York, 1991).
[47] See, for example, A. Abragam, Principles of Nuclear Magnetic Resonance (Oxford University Press, New York, 1961).

[48] H. Barnum, M. A. Nielsen, and B. Schumacher, Phys. Rev. A 57, 4153 (1998).

[49] K. Kraus, Ann. Phys. (N.Y.) 64, 311 (1971).

[50] Y. Kondo, Y. Matsuzaki, K. Matsushima, and J. G. Filgueiras, New J. Phys. 18, 013033 (2016).

[51] K. Hornberger, Lect. Notes Phys. 768, 221 (2009).

[52] G. Lindblad, Commun. Math. Phys. 48, 119 (1976).

[53] F. Brito and T. Werlang, New J. Phys. 17, 072001 (2015).

[54] I. Almog, Y. Sagi, G. Gordon, G. Bensky, G. Kurizki, and N. Davidson, J. Phys. B 44, 154006 (2011); G. A. Álvarez and D. Suter, Phys. Rev. Lett. 107, 230501 (2011); T. Yuge, S. Sasaki, and Y. Hirayama, ibid. 107, 170504 (2011).

[55] J. Bylander, S. Gustavsson, F. Yan, F. Yoshihara, K. Harrabi, G. Fitch, D. G. Cory, Y. Nakamura, J. Tsai, and W. D. Olive, Nat. Phys. 7, 565 (2011).

[56] N. Bar-Gill, L. M. Pham, A. Jarmola, D. Budker, and R. L. Walsworth, Nat. Commun. 4, 1743 (2013).

[57] C. Addis, F. Ciccarello, M. Cascio, G. M. Palma, and S. Maniscalco, New J. Phys. 17, 123004 (2015).

[58] T. Gullion, D. B. Baker, and M. S. Conradi, J. Magn. Reson. 89, 479 (1990).

[59] G. A. Álvarez, A. Ajoy, X. Peng, and D. Suter, Phys. Rev. A 82, 042306 (2010); A. M. Souza, G. A. Álvarez, and D. Suter, ibid. 85, 032306 (2012); M. A. Ali Ahmed, G. A. Álvarez, and D. Suter, ibid. 87, 042309 (2013).

[60] S. Simmons, J. A. Jones, S. D. Karlen, A. Ardavan, and J. J. L. Morton, Phys. Rev. A 82, 022330 (2010).

[61] Z. H. Wang, W. Zhang, A. M. Tyryshkin, S. A. Lyon, J. W. Ager, E. E. Haller, and V. V. Dobrovitski, Phys. Rev. B 85, 085206 (2012).

[62] E. Hosseini-Lapaser, K. Maruyama, D. Burgarth, T. Takui, Y. Kondo, and M. Nakahara, New J. Phys. 14, 013043 (2012)

[63] Y. Matsuzaki, S. C. Benjamin, and J. Fitzsimons, Phys. Rev. A 84, 012103 (2011).

[64] A. W. Chin, S. F. Huelga, and M. B. Plenio, Phys. Rev. Lett. 109, 233601 (2012).

[65] S. F. Huelga, C. Macchiavello, T. Pellizzari, A. K. Ekert, M. B. Plenio, and J. I. Cirac, Phys. Rev. Lett. 79, 3865 (1997).

[66] S. Saito, X. Zhu, R. Amsüss, Y. Matsuzaki, K. Kakuyanagi, T. Shimo-Oka, N. Mizuochi, K. Nemoto, W. J. Munro, and K. Semba, Phys. Rev. Lett. 111, 107008 (2013).

[67] Y. Kubo, C. Grezes, A. Dewes, T. Umeda, J. Isoya, H. Sumiya, N. Morishita, H. Abe, S. Onoda, T. Ohshima, V. Jacques, A. Dréau, J.-F. Roch, I. Diniz, A. Auffeves, D. Vion, D. Esteve, and P. Bertet, Phys. Rev. Lett. 107, 220501 (2011).

[68] S. Gustavsson, F. Yan, J. Bylander, F. Yoshihara, Y. Nakamura, T. P. Orlando, and W. D. Oliver, Phys. Rev. Lett. 109, 010502 (2012).

[69] P. C. Maurer, G. Kucsko, C. Latta, L. Jiang, N. Y. Yao, S. D. Bennett, F. Pastawski, D. Hunger, N. Chisholm, M. Markham, D. J. Twitchen, J. I. Cirac, and M. D. Lukin, Science 336, 1283 (2012).

[70] L. Müler, A. Kumar, T. Baumann, and R. R. Ernst, Phys. Rev. Lett. 32, 1402 (1974); G. A. Álvarez, E. P. Danieli, P. R. Levstein, and H. M. Pastawski, J. Chem. Phys. 124, 194507 (2006). 